\title{
Generation of copper nanoparticles by electro-exploding wire technique for various pressures of the surrounding medium.
}

\author{
Rashmita Das, Basanta Kumar Das, M. V. Suryanarayana, M. Sankari, \\ Archana Sharma \\ Pulse Power \& Electromagnetics Division, Bhabha Atomic Research Centre, Autonagar, Visakhapatnam- \\ 530012, India \\ Key words: Copper nanoparticles, Electro -exploding wire, TEM, Particle size distribution, Optical microscopy, \\ $X R D$
}

\begin{abstract}
This paper presents the experimental results on explosion of copper wire at a discharge voltage of $9 \mathrm{kV}$ for the generation of $\mathrm{Cu}$ nanoparticles in nitrogen environment at different pressures. The current and voltage waveforms for different pressures of the nitrogen gas have been recorded by a current transformer and a high voltage probe. Nanoparticles generated by the electro exploded wire method were characterized by both optical microscopy and transmission electron microscopy. Experiments show that the variation of pressure of nitrogen gas inside the exploding chamber determines the size of the particles formed during the explosion of the copper wire. It is observed that for a pressure of less than 230 mbar of nitrogen gas, the wire does not explode and the copper wire remains intact. Micro particle generation occurred at a pressure of $2.3 \times 10^{2} \mathrm{mbar}$ of nitrogen gas and nanoparticles were formed when the pressure was further increased to $1 \times 10^{3}$ mbar. Experimental results show that we could deposit maximum energy into the metal core when the wire was exploded by working in the high pressure regime of the nitrogen gas resulting in the production of nanoparticles. Transmission electron microscopy reveals the size of the nanoparticles to be in the range of 35 to $75 \mathrm{~nm}$.
\end{abstract}

\section{INTRODUCTION}

Nanotechnology has been widely used because of its capability to change the structure of molecules at an atomic level. Metal nanoparticles have different or superior properties than the bulk material. It is well known that the shape, size and micro structures are the main factors in determining the properties of nanoparticles. This article deals with the explosion of metallic wire to produce copper nanoparticles as a simple, low cost and environment friendly method which can then be used for broad spectrum of applications in photonics, chemical sensors, biological sensors, in fuel cells, pigments, integrated circuit substrate formation, ink jet printing of electronic lines and components etc.

Some of the specific application of copper nanoparticles is in the production of high strength metal and alloys, used as heat sinks, for the production of highly thermally conductive material, and also as an efficient catalyst. Conductive inks and pastes containing copper nanoparticles can be used as a substitute for very expensive noble metals used in printed electronics, displays and also in transmission and conductive thin film applications. The control over particle size is highly desirable because size dependent properties of copper nanoparticles are expected to provide additional applications. There are various top down and bottom up methods to produce copper nanoparticles. In this work we have studied the effect of pressure of the surrounding medium on the production of copper nanoparticles by the exploding wire method.

Exploding wire exhibits numerous applications in the production of metal nanoparticles [1-4], wire array Z-pinches [5-6], fuses, in the inductive energy storage [7] and in other areas too. Therefore, extensive understanding of the underlying physical phenomena in exploding wire method is important for its effective utilization. In the process of production of copper nanoparticles, there is a significant effect of pressure on the process of wire explosion. In this paper, we describe the transition of the copper wire during explosion from solid state through a dispersion of micro particles and finally a fine dispersion of nanoparticles under different pressures of nitrogen gas. Research in the field of nanoparticles has gone up by leaps and bounds due to the development of advanced tools to synthesize and characterize the material. The synthesis of nanoparticles depends upon the basic understanding of nanoparticles physics and chemistry and the subsequent commercialization of the same. Basically the production of nanoparticles is of two types, top down and bottom up method. The former decrease the size of the material to nano scale and latter starts the development from atomic scale. Wire explosion is basically a mixed top-down and bottom up approach to produce metallic nano-powders. A 
pulsed discharge system is used to supply a high power pulsed current to a thin metal wire and this lead to the explosion of the wire. Large amount of heat from Joule heating is dissipated into the wire. The wire melts, evaporates and subsequently ionizes the atoms. Plasma formed during the process expands due to its high temperature and high density. This plasma is rapidly cooled during expansion when it interacts with the surrounding gas and nanoparticles are formed through nucleation process. Generally the wire explosion technique has several advantages for the generation of nanoparticles. Firstly, the energy deposition can be controlled precisely, and secondly, the discharge energy is deposited into the wire with high efficiency and the wire is exploded rapidly to form a supersaturated vapor. Secondly, since no other chemicals are used in the process, pure $\mathrm{Cu}$ nanoparticles can be produced. Studies showed that the size, morphology, stability and properties of the nanoparticles depend upon the experimental conditions. Hence the design and optimization of the nanoparticles synthesis in order to control the size, morphology and stability of the nanoparticles is a very interesting field and has drawn huge attention from researchers in this field. In this paper we discuss the effect of pressure of the surrounding medium on the formation of nanoparticles in the electro exploding wire technique.

\section{EXPERIMENTAL TECHNIQUE}

Schematic circuit diagram for nanopowder production using the exploding wire method is shown in Figure-1. The details of the experimental set up are described in reference [8]. All the experimental parameters are tabulated in table-1.In the exploding wire method, a $7.1 \mu \mathrm{F}$ capacitor was charged to a voltage of $9 \mathrm{kV}$ and then the energy of the capacitor was discharged into a copper wire of diameter of $0.26 \mathrm{~mm}$. The diameter of the wire was obtained from the action integral of the copper material. This is calculated from the equation given below.

$$
\frac{1}{s^{2}} \int_{0}^{t_{b}} I(t)^{2} d t=\gamma \int_{e_{0}}^{e_{v}} \frac{1}{\rho(e)} d e=a
$$

$\mathrm{I}(\mathrm{t})$ is the current flowing in the conductor, $\rho$ is the resistivity of the material, ' $s$ ' is the cross section of the wire, $\gamma$ is the density of the material, $\mathrm{e}$ is the internal energy per unit mass of the material.

Action of copper $(a)$ is $1.31 \times 10^{17} \mathrm{~A}^{2} \mathrm{sec} \mathrm{m}^{-}$

4 . For explosion of the wire, the action of the system should be nearly equal to the required action of copper. The detailed calculation of the diameter of the copper wire from the action integral of copper is described in [9]. The exploding wire experiment was carried out in a cylindrical SS chamber with the provision to install the wire straight and various ports have been provided for the injection of the gas, pumping port for connection to the vacuum pump and port for vacuum measurement. For each set of experiment the pressure was varied to investigate the actual size distribution of the exploded material. The pressure in the experimental chamber was varied from $4.2 \times 10^{-2}$ mbar to atmospheric pressure with nitrogen. The current was measured using a current transformer (CT), while the voltage was measured using a voltage divider. The typical current and voltage waveforms are shown in figures $2,3 \& 4$. The energy deposited into the wire was calculated from voltage and current curves and is shown in figure 5. One clean circular Mylar sheet whose diameter was of almost equal to that of the chamber was placed inside the experimental chamber just below the installed wire. This sheet was chosen so that nanoparticles collection will be simple and will not stick to the substrate. The collected sample was taken for characterization with utmost care to avoid exposure to air. The size of the particles obtained from the explosion of the wire was measured with optical microscope as well as with transmission electron microscope (TEM). The $\mathrm{Cu}$ nanoparticles produced were dispersed into acetone for preparing the sample to be analyzed by TEM. Particle size distributions were determined from multiple TEM images by manual counting. Images from optical microscope and TEM are shown in figures 6 and 7. The sample was also characterized using $\mathrm{X}$-ray diffraction (XRD) by powder diffraction method using the $\mathrm{K}_{\alpha}$ line of copper $(\lambda=0.15405 \mathrm{~nm})$, the spectrum is shown in figure 8 . XRD indicates the presence of pure copper in the sample. Particle distribution of exploded nanoparticles is shown in figure 9. Particle distribution has been derived from multiple images of TEM. The average size of the particle is $45 \mathrm{~nm}$ with a standard deviation of 7 .

Mass of the exploded copper wire was $57 \mathrm{mg}$. It was exploded in an enclosed SS cylindrical pressure vessel with Mylar sheet as liner for sample collection. The powder collected on the sheet placed on the bottom of the chamber was collected for characterization. During explosion the powder was also deposited on the walls of the chamber, therefore it was not possible to collect the entire powder obtained from the explosion. Further work towards the quantification of the nanoparticles is under progress. But it is sure from the TEM image that all the particles are of size less than $100 \mathrm{~nm}$.

\section{RESULTS AND DISCUSSION}

It is realized that energy deposited to the conductor and duration of current flow have major impact on particles produced by this process [6]. For each set of experiment the capacitor $(7.1 \mu \mathrm{F})$ was charged to a charging voltage of $9 \mathrm{kV}$. Current and voltage waveform were recorded for pressure values of $4.2 \times 10^{-2}$ mbar to $10^{3}$ mbar. Peak current measured was around $20 \mathrm{kA}$, however peak voltages varied for each 
pressure value. The energy deposition was measured from the current and voltage waveform. Current and voltage waveform for $4.2 \times 10^{-2} \mathrm{mbar}, 2.3 \times 10^{2} \mathrm{mbar}$ and $10^{3}$ mbar are shown in figures 2 - 4. Transition of the copper wire from solid through micro particle dispersion and finally to nanoparticles dispersion is tabulated in Table-2. Vaporization energy required for the wire of length $12 \mathrm{~cm}$ and diameter of $0.26 \mathrm{~mm}$ is 207J. Ringing behavior of current waveform shown in figure 2 and 3 is due to lesser amount of energy that is deposited into the wire which keeps the path between the two electrodes close and behaves as a shorted LC system. As seen from figure-4 most of the deposited energy has gone for current interruption. Energy deposited into the wire at $4.2 \times 10^{-2} \mathrm{mbar}$ amounts to 120 Joule which is not sufficient even to melt the wire and it had gone for the breakdown of the surrounding gas medium. When the pressure was increased to 2.3 $\times 10^{2}$ mbar, the energy deposited into the wire was 150 Joule and the wire exploded producing micro size particles. The particle size was studied using optical microscope. The image of the optical microscope is shown in figure-6. For pressure of $10^{3} \mathrm{mbar}$, energy deposited was highest i.e, 250 Joule. Under these experimental conditions the explosion of wire occurred and the wire disintegrated into nanoparticles. The image of the nanoparticles observed by TEM is shown in figure-7.

Due to joule heating, the temperature of the conductor rises followed by melting and heating of the wire which is in the liquid phase. Before transition to the gas phase the liquid goes through the boiling point and superheating. After the evaporation of the wire, the plasma deposited vapor liquid mixture begins to expand in the medium due to enormous difference in temperature and pressure between the conductor vapor and the ambient gas. The vapor particles formed during the vaporization process get cooled by collision with atoms in the ambient which are at room temperature. The temperature of the vapor particles rapidly gets cooled to a value much lower than the freezing point of the metal gas vapor thereby reaching a super cooled phase which creates stable equilibrium by generation of new particles through nucleation. There are two types of nucleation.

a. Homogeneous nucleation: New particles are formed from supersaturated vapor species in the absence of any condensable sites.

b. Heterogeneous nucleation: Vapor particles nucleate over any particle already existing. In exploding wire method in the rough vacuum domain, there are condensable sites, so particles are formed through heterogeneous nucleation. After nucleation of particles, coagulation of particles take place as the particle collides with each other due to Brownian motion.

Since copper oxidizes in air, the explosion of wire was carried out in an inert atmosphere like nitrogen gas. The copper nitrogen bond formed during explosion does not stay long, it breaks producing pure copper nanoparticles. XRD data as shown in figure- 8 reveals the presence of pure copper crystal. The average particle size as shown in figure-9, obtained due to explosion of copper wire in nitrogen pressure of 1000 mbar is $45 \mathrm{~nm}$.

Energy deposited in the wire is limited by the plasma formation which in turn depends on the pressure of the surrounding medium. Resistance in the medium comes due to collision of the molecules and is less for lower pressure of the surrounding gas. When pressure of the gas is low around the wire, the applied voltage breaks down the gas. Since the wire is the more resistive path and the current chooses the low resistance path which is the medium so discharge breakdown of the medium occurs. For higher pressures the wire works as a low resistive path and energy deposition is more in the wire.

Micrometer size particles originated from liquid droplet could be effectively eliminated by heating the wire rapidly and suppressing the formation of plasma. The relationship between plasma density and energy deposition in to the wire is studied and reported in Ref [10]. This could be achieved by increasing the $\mathrm{dI} / \mathrm{dt}$ which could be achieved by increasing the voltage applied to the wire while keeping the inductance to as low value as possible. But higher voltage led to the breakdown of the gas medium. Break down of the gas medium could be prevented by increasing the gas pressure around the wire.

\section{CONCLUSIONS}

Our measurements confirm that copper nanoparticles have been generated by electro exploding wire technique. A change of the wire surrounding medium in the explosion process presents a simple and flexible technique to modify the properties of the nanoparticles. The TEM images show that the synthesized nanoparticles have a narrow particle size distribution in the range of 35 to $75 \mathrm{~nm}$ with an average particle size of $45 \mathrm{~nm}$.

Effect of nitrogen pressure has a significant role for the production of pure copper nanoparticles using electro explosion wire technique of copper wire. At a nitrogen pressure of one atmosphere, the energy deposited into the wire is maximum. But the energy deposited decreases with decrease in the gas pressure as the energy goes into the breakdown of the gas. Electrical glow discharge creates the shortest path between the electrodes and is seen as ringing in the current waveform. At sub atmospheric pressures, for a particular combination of electrode gap and applied voltage, electrical break down of the gas occurs and is attributed to the electrical glow discharge regime. Purity of the copper nanoparticles produced in presence of nitrogen is due to the 
unstable bonding between copper and nitrogen. Hence copper wire explosion at one atmospheric pressure of nitrogen at $9 \mathrm{KV}$ charging voltage for a capacitor of $7.1 \mu \mathrm{F}$ could produce copper nanoparticles with an average size of $45 \mathrm{~nm}$.

\section{REFERENCES}

[1] Abdullah Alqudami, SaqrMunassar, Nabil Alhemiary, "The sorption properties of exploding wire prepared $\mathrm{Ag}, \mathrm{Fe}$ and $\mathrm{ZnO}$ nanoparticles for nitrite removal from water" International Journal of Material Science (IJMSCI) Volume 3 Issue 1 (2013)

[2] Rashmita Das, Basanta Kumar Das and Anurag Shyam "Synthesis and characterization of copper nanoparticles by using the Exploding Wire Method" Journal of the Korean Physical Society, Vol. 61, No. 5, pp. 710 - 712 (2012)

[3] T. K. Sindhu, R.Sarathi, S. R. Chakravarthy, "Generation and characterization of nano aluminium powder obtained through wire explosion process" Bulletin of Materials Science 2007,30,187-195

[4] Y. S. Lee, B. Bora, S. L. Yap, C. S. Wong, "Effect of ambient air pressure on synthesis of copper and copper oxide nanoparticles by wire explosion process", Current Applied Physics. 12 (2012) 199-203

[5] Burdiak, G. C.; Lebedev, S. V.; Hall, G. N.; Harvey-Thompson, A. J.; Suzuki-Vidal, F.; Swadling, G. F.; Khoory, E.; Pickworth, L.; Bland, S. N.; de Grouchy, P.; Skidmore, J, "Determination of the inductance of imploding wire array Z-pinches using measurements of load voltage"Physics of Plasmas, Volume 20, Issue 3, pp. 032705032705-8 (2013).

[6] K.N. Mitrofanov, E.V. Grabovski, A.N. Gritsuk, Ya.N. Laukhin, V.V. Aleksandrov, G. Moleinik, S.F. Medovshchikov and A.P. Shevelko "Specific features of the structure of the Z-pinch emitting region formed during the implosion of a foam-wire load at the Angara-5-1 facility" Plasma Physics Reports, Volume 39, No.1, pp. 62-85 (2013)
[7] Kolacek, K. Schmidt, J. Prukner, V. Ways to discharge-based soft X-ray lasers with the wavelength $<15 \mathrm{~nm}$, Laser and Particle Beams 26, 2008, 167-178

[8] R. Das, B. Das, R. Shukla, P. Deb, T, Prabaharan and A. Shyam "Experimental study of exploding wire method for production of metal nanoparticles" Instruments and Experimental Techniques, Vol.55, No.1, PP.131-133 (2012)

[9] Rashmita Das, Basanta Kumar Das, Rohit Shukla, T. Prabaharan and Anurag Shyam "Analysis of Electrical Explosion of Wire systems for the production of nanopowder "Sadhana, 37 (5), 629-635 (2012)

[10] Junping Zhao, Zhuo Xu, Wenyu Yan, Haoyu Liu, and Qiaogen Zhang. "Characteristics and Diffusion of Electrical Explosion Plasma of Aluminum Wire in Argon Gas" IEEE TRANSACTIONS ON PLASMA SCIENCE, VOL. 45, NO. 2, FEBRUARY 2017

\section{FIGURE CAPTION}

Figure.1: Schematic diagram of the exploding wire circuit.

Figure. 2: Voltage and Current waveform at $4.2 \times 10^{-2}$ mbar of nitrogen gas

Figure. 3: Voltage and Current waveform at $2.3 \times 10^{2}$ mbar of nitrogen gas

Figure. 4: Voltage and Current waveform at $10^{3} \mathrm{mbar}$ of nitrogen gas

Figure. 5: Energy deposited into the wire during wire explosion for various pressures of nitrogen gas

Figure. 6: Optical microscope image of exploded material at $2.3 \times 10^{2}$ mbar of nitrogen gas pressure.

Figure. 7: Transmission electron microscope image of exploded material at 1 bar of nitrogen gas pressure Figure. 8: XRD spectrum for exploded material Figure.9: Particle size distribution of exploded material at 1 bar of nitrogen gas pressure 


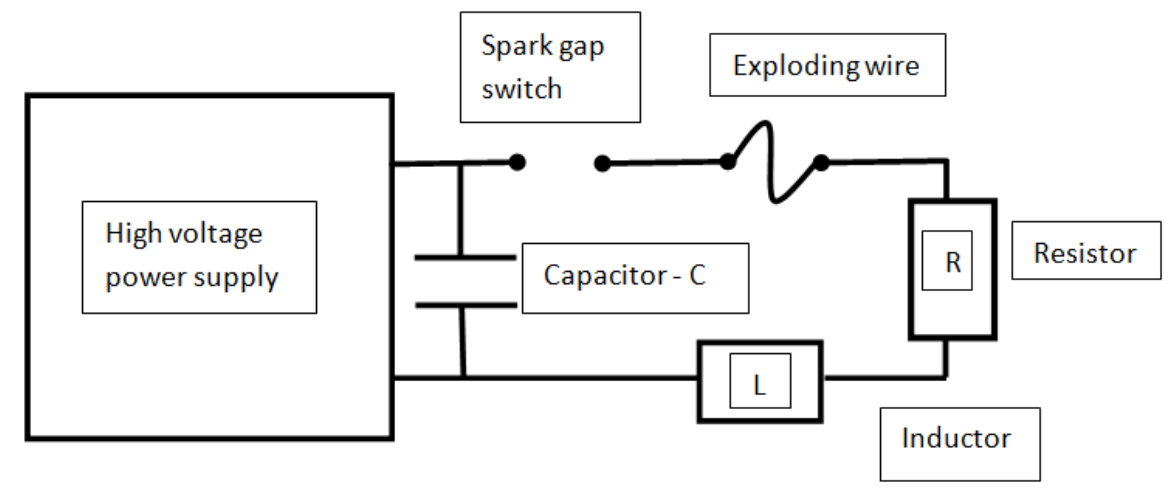

Figure.1: Schematic diagram of the exploding wire circuit.

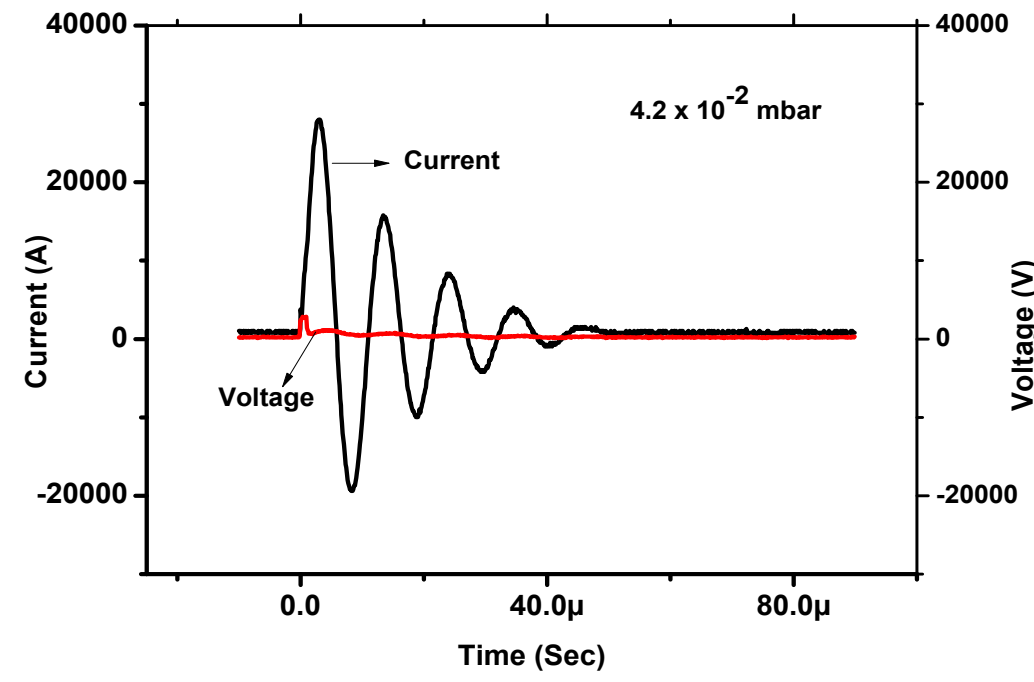

Figure.2: Voltage and current waveform at $4.2 \times 10^{-2} \mathrm{mbar}$ of nitrogen gas.

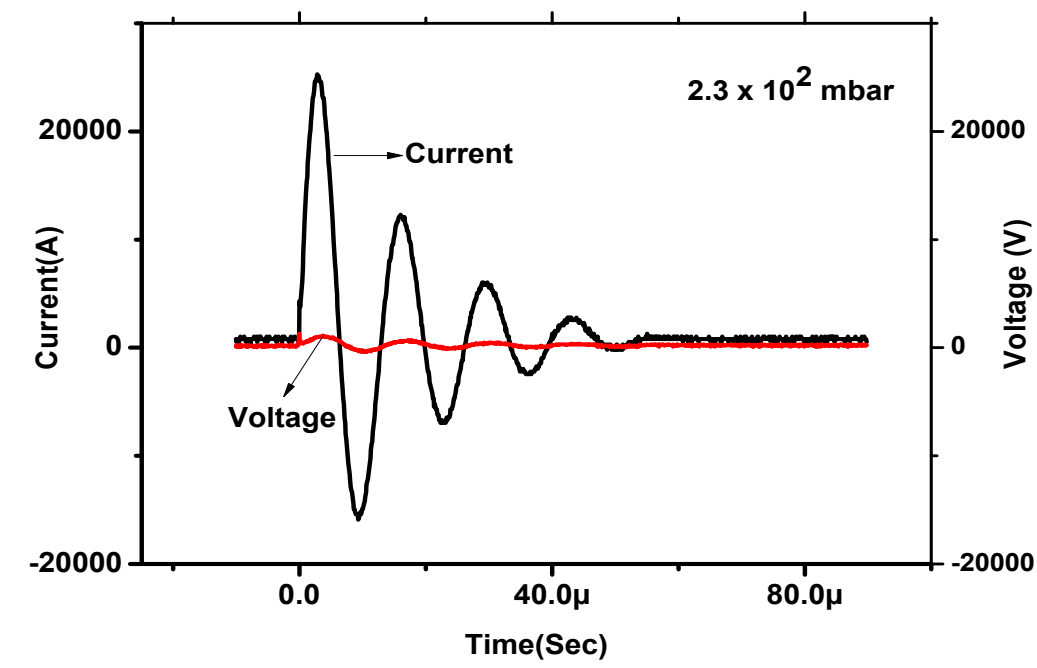

Figure.3: Voltage and current waveform at $2.3 \times 10^{2} \mathrm{mbar}$ of nitrogen gas. 


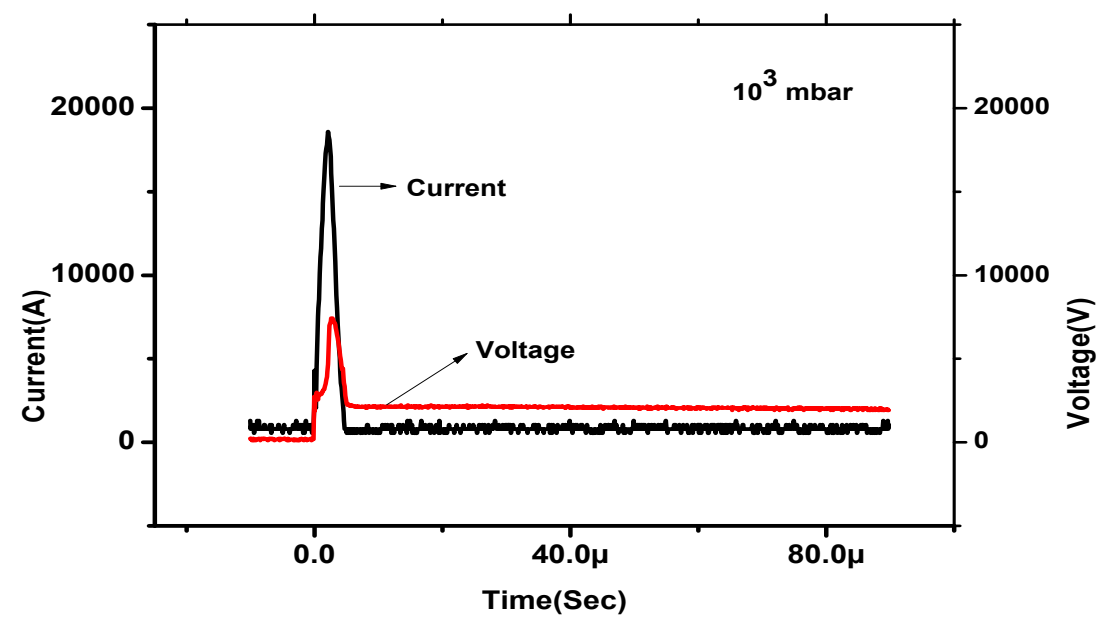

Figure.4: Voltage and current waveform at $10^{3}$ mbar of nitrogen gas.

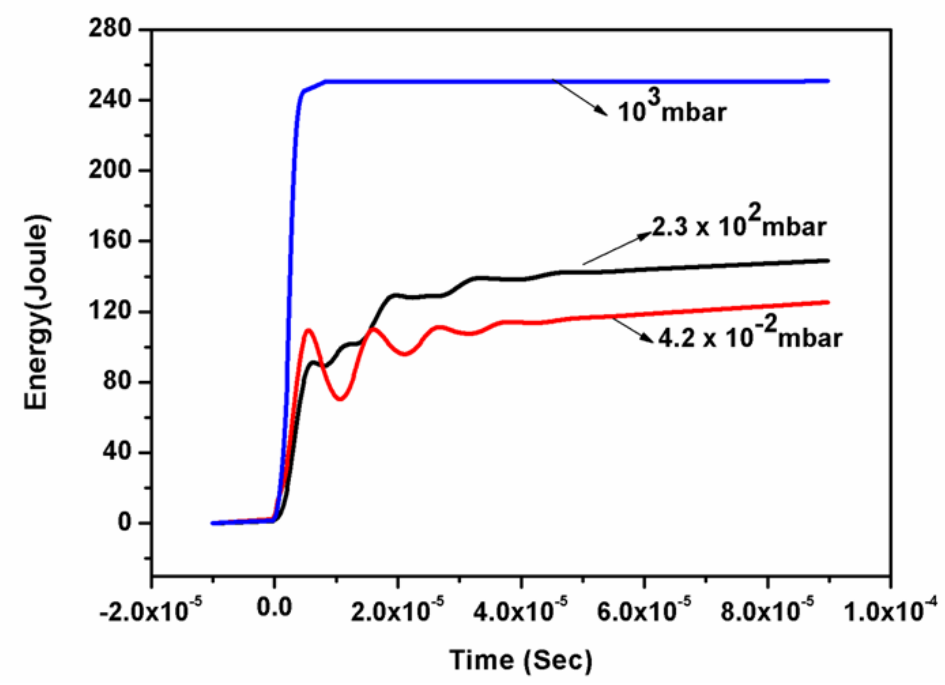

Figure.5: Energy deposited into the wire during wire explosion for various pressures of nitrogen gas.

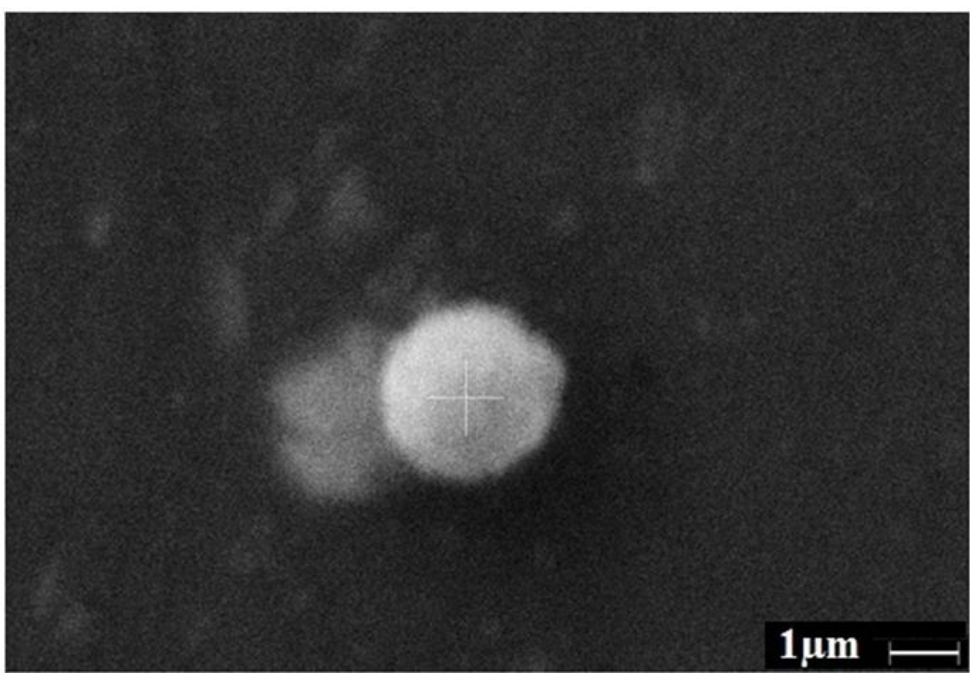

Figure.6: Optical microscope image of exploded material at $2.3 \times 10^{2}$ mbar of nitrogen gas pressure. 


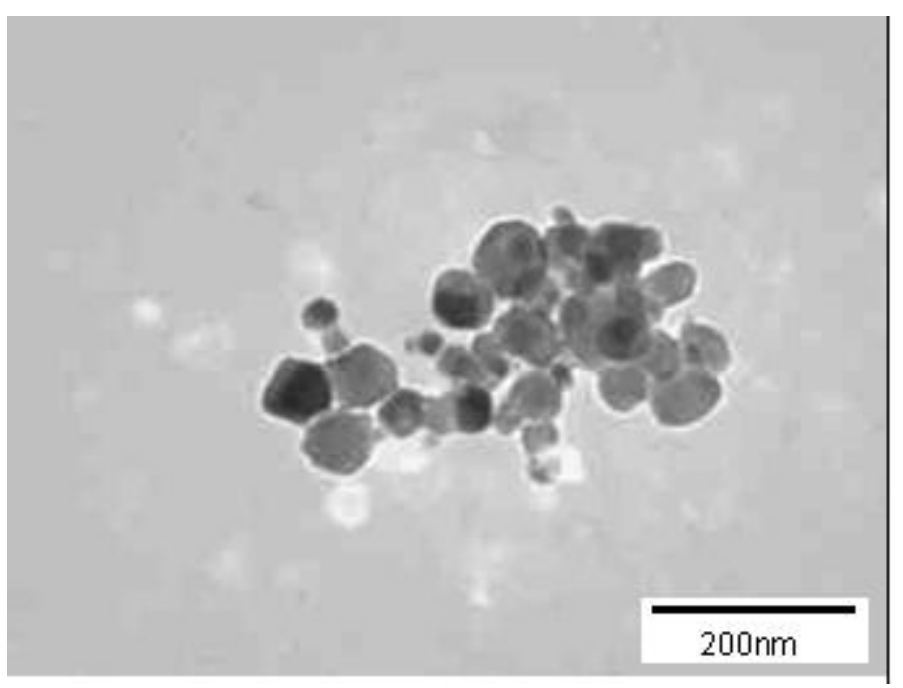

Figure.7: Transmission electron microscope image of the exploded material at $1 \mathrm{bar}$ of nitrogen gas pressure.

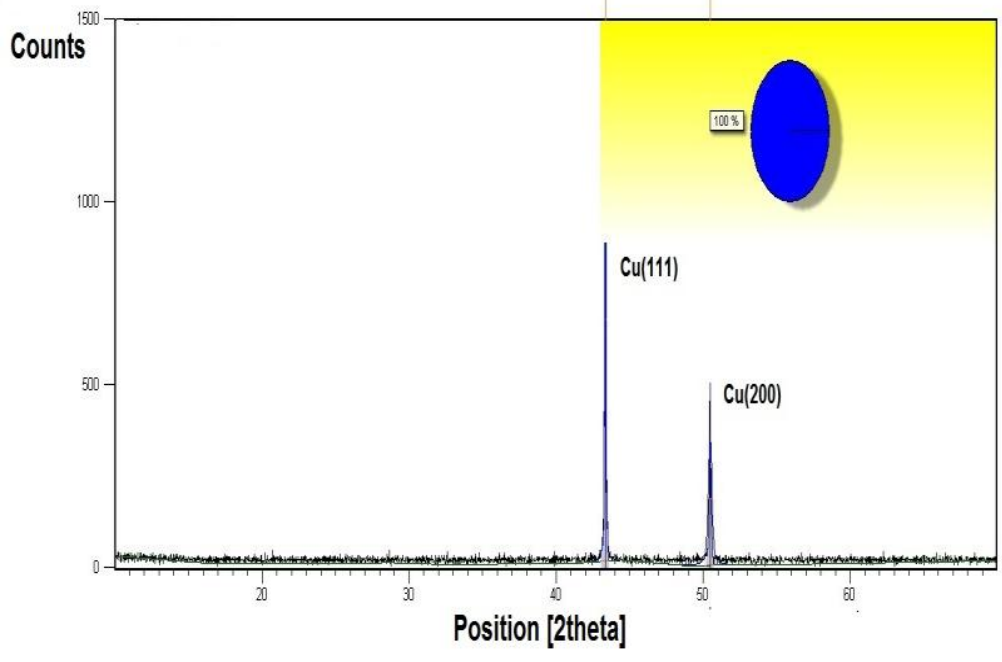

Figure.8: XRD spectrum for the exploded material

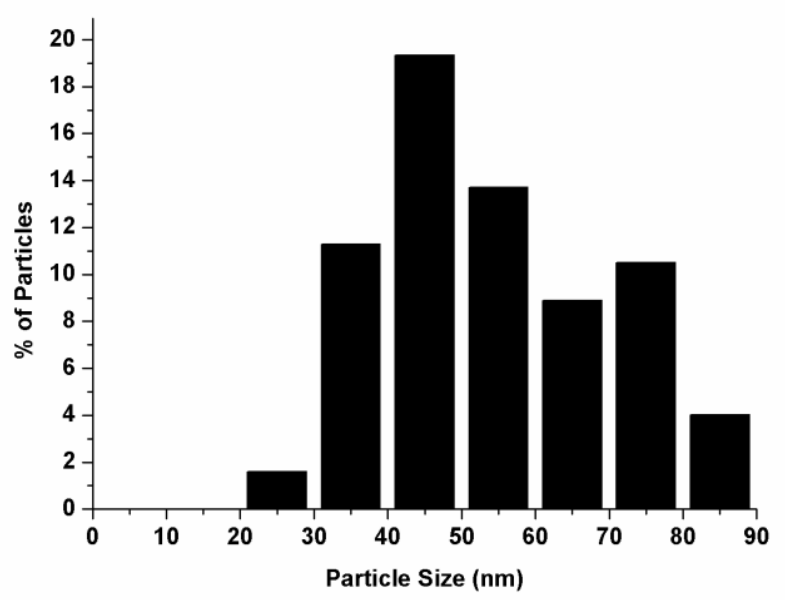

Figure.9: Particle size distribution of the exploded copper nanoparticles at 1 bar of nitrogen gas pressure. 
Table 1: Table of the values of the experimental parameters of the exploding wire setup.

\begin{tabular}{|c|c|c|}
\hline SI no: & Parameter & Value \\
\hline 1 & Capacitance & $7.1 \mu \mathrm{F}$ \\
\hline 2 & Inductance & $700 \mathrm{nH}$ \\
\hline 3 & Charging voltage & $9 \mathrm{kV}$ \\
\hline 4 & CT & $(1: 1000) \mathrm{V} / \mathrm{A}$ \\
\hline 5 & HV probe & Nitrogen \\
\hline 6 & Surrounding gas medium & $1 \mathrm{bar}$ \\
\hline 7 & Pressure & $0.26 \mathrm{~mm}$ \\
\hline 8 & Diameter of the wire & $12 \mathrm{~cm}$ \\
\hline 9 & Length of the wire & \\
\hline
\end{tabular}

Table 2: Transition of the copper wire from solid through micro particle dispersion and finally to nanoparticles.

\begin{tabular}{|l|l|l|}
\hline Pressure (mbar) & Physical state of the copper wire & Deposited Energy (Joule) \\
\hline $4.2 \times 10^{-2}$ & Not broken & 120 \\
\hline $2.3 \times 10^{2}$ & Micro particles & 150 \\
\hline $1 \times 10^{3}$ & Exploded and nanoparticles & 250 \\
\hline
\end{tabular}

